\title{
Space-Varying Iterative Restoration of Diffuse Optical Tomograms Reconstructed by the Photon Average Trajectories Method
}

\author{
Alexander B. Konovalov, ${ }^{1}$ Vitaly V. Vlasov, ${ }^{1}$ Olga V. Kravtsenyuk, ${ }^{2}$ and Vladimir V. Lyubimov ${ }^{3}$ \\ ${ }^{1}$ Russian Federal Nuclear Centre, Institute of Technical Physics, P.O. Box 245, \\ Snezhisk Chelyabinsk Region 456770, Russia \\ ${ }^{2}$ Institute of Electronic Structure and Laser, Foundation for Research and Technology - Hellas, \\ P.O. Box 1527, Vassilika Vouton, Heraklion 71110, Greece \\ ${ }^{3}$ Research Institute for Laser Physics, 12 Birzhevaya Lin, Saint Petersburg 199034, Russia
}

Received 2 February 2006; Revised 2 August 2006; Accepted 29 October 2006

Recommended by Lisimachos Paul Kondi

The possibility of improving the spatial resolution of diffuse optical tomograms reconstructed by the photon average trajectories (PAT) method is substantiated. The PAT method recently presented by us is based on a concept of an average statistical trajectory for transfer of light energy, the photon average trajectory (PAT). The inverse problem of diffuse optical tomography is reduced to a solution of an integral equation with integration along a conditional PAT. As a result, the conventional algorithms of projection computed tomography can be used for fast reconstruction of diffuse optical images. The shortcoming of the PAT method is that it reconstructs the images blurred due to averaging over spatial distributions of photons which form the signal measured by the receiver. To improve the resolution, we apply a spatially variant blur model based on an interpolation of the spatially invariant point spread functions simulated for the different small subregions of the image domain. Two iterative algorithms for solving a system of linear algebraic equations, the conjugate gradient algorithm for least squares problem and the modified residual norm steepest descent algorithm, are used for deblurring. It is shown that a $27 \%$ gain in spatial resolution can be obtained.

Copyright $\odot 2007$ Hindawi Publishing Corporation. All rights reserved.

\section{INTRODUCTION}

The main problem of medical diffuse optical tomography (DOT) is the low spatial resolution due to multiple light scattering, which causes photons to propagate diffusely in a tissue. To reconstruct diffuse optical tomograms with best resolution, "well-designed" methods such as Newton-like and gradient-like ones [1-3], which use exact forward models, are generally applied. These methods belong to a class of a so-called "multistep" techniques, as the weighting matrix of equation system is updated on each iteration of the solution approximation. They require computation time not less than a few minutes for 2D image reconstruction and consequently are inapplicable for real-time medical explorations. Over the past few years, we have presented a new DOT method [4-16] based on a concept of an average statistical trajectory for transfer of light energy, the photon average trajectory (PAT). The essence of this concept is in rep- resenting the process of the photon energy transport from a source to a receiver in a form admitting probabilistic interpretation. By this method, the inverse problem of DOT is reduced to a solution of an integral equation with integration along a conditional PAT that is curvilinear in the common case. As a result, the PAT method can be implemented as a "one-step" technique with the use of fast algorithms of projection computed tomography and can considerably save the computation time. Our experience shows that not only the algebraic techniques $[11,16]$ but also the real-time filtered backprojection algorithm (FBP) [12-15] can be successfully applied to reconstruct the internal region of the object, where the PATs tend to the straight line. The shortcoming of the PAT method is that it reconstructs the tomograms blurred due to averaging over spatial distributions of photons which form the signal measured by the receiver. To improve the spatial resolution, we have tried to use FBP with special filtration of shadows (Vainberg [12-15] or hybrid Vainberg-Butterworth 
filtration [15]). This algorithm gives a 20\%-gain in resolution but does not correctly restore the inhomogeneity profile as the averaging kernel is not taken into account. A profile is reconstructed with a typical incline distinctly visible for any inhomogeneity remote well away from the object center. In present paper, we consider an alternative way of enhancing the resolution, based on the post-reconstruction restoration of the diffuse optical tomograms. We show that the blur due to averaging over distributions of diffusive photons is described with the point spread function (PSF) strongly variant against spatial shift. Therefore, a spatially variant blur model should be applied for PAT image restoration. We assume the blur model recently developed by Professor Nagy and his colleagues [17-19]. It is described by a system of linear algebraic equations and based on the assumption that in small subregions of the image domain, the PSF is essentially spatially invariant. To form the matrix modeling the blurring operation, the invariant PSFs corresponding to subregions are sewn together with an interpolation approach. Then standard iterative algorithms for solving a system of linear algebraic equations are used to calculate the true image. To study the efficiency of the blur model assumed, a numerical experiment on reconstruction of circular scattering objects with absorbing inhomogeneities is conducted, the individual PSFs are simulated for different subregions of the image domain, the weighting matrix that models the blurring operation is formed, and two well-known iterative algorithms for solving a system of linear algebraic equations are applied to restore the reconstructed blurred tomograms. These algorithms are the conjugate gradient algorithm for least squares problem (CGLS) [20] and the modified residual norm steepest descent algorithm (MRNSD) $[21,22]$. We show below that both of them allow a good gain in spatial resolution to be achieved without visible distortions of the image profile. In number, this gain is estimated by means of the modulation transfer function (MTF) and seems to be greater than that obtained by using FBP with Vainberg filtration.

\section{RECONSTRUCTION OF BLURRED TOMOGRAMS}

\subsection{Fundamental equation of the PAT method}

The PAT method is based on a probabilistic interpretation of the photon migration process with description by means of statistical characteristics. The introduction of such characteristics as the PAT and the average velocity of the photon movement allows a relative shadow caused by optical inhomogeneities to be connected with a function of the object inhomogeneity distribution through a curvilinear integral along the PAT, analogue of the Radon transform.

Let the photons migrate in a strongly scattering media from a source space-time point $(0,0)$ to a receiver space-time point $(\mathbf{r}, t)$. A relative contribution of photons located at an intermediate space-time point $\left(\mathbf{r}_{1}, \tau\right)$ to the value of photon density at $(\mathbf{r}, t)$ can be characterized by a conditional probability density [4-8]:

$$
P\left(\mathbf{r}_{1}, \tau ; \mathbf{r}, t\right)=\frac{P\left(\mathbf{r}_{1}, \tau\right) P\left(\mathbf{r}-\mathbf{r}_{1}, t-\tau\right)}{P(\mathbf{r}, t)},
$$

where $P(\mathbf{r}, t)$ is a probability density of the photon migration from $(0,0)$ to $(\mathbf{r}, t)$. If the photon density $\varphi(\mathbf{r}, t)$ satisfies the time-dependent diffusion equation for a volume $V$ with a limited piecewise-closed smooth surface for an instantaneous point source and the Robin boundary condition [23], the probability density $P\left(\mathbf{r}_{1}, \tau ; \mathbf{r}, t\right)$ is expressed as [11]

$$
P\left(\mathbf{r}_{1}, \tau ; \mathbf{r}, t\right)=\frac{\varphi\left(\mathbf{r}_{1}, \tau\right) G\left(\mathbf{r}-\mathbf{r}_{1}, t-\tau\right)}{\int_{V} \varphi\left(\mathbf{r}_{1}, \tau\right) G\left(\mathbf{r}-\mathbf{r}_{1}, t-\tau\right) d^{3} r_{1}},
$$

where $G(\mathbf{r}, t)$ is the Green function. The first statistical moment

$$
\mathbf{R}(\mathbf{r}, t, \tau)=\int_{V} \mathbf{r}_{1} P\left(\mathbf{r}_{1}, \tau ; \mathbf{r}, t\right) d^{3} r_{1}
$$

as a function of time $\tau$, describes the trajectory of the mass center of the photon distribution, namely, the PAT. Correspondingly, the velocity of the mass center is given by the expression

$$
v(\tau)=\left|\frac{d \mathbf{R}(\tau)}{d \tau}\right| .
$$

It is seen from (2)-(4) that characteristics (3) and (4) can be analytically calculated only for objects of quite simple forms. For complex geometries, some approximations must be made.

Let us define a relative shadow $g$ as a logarithm of the relation between the value of the signal intensity $I$, caused by presence of the inhomogeneities and the value of unperturbed signal intensity $I_{0}$, measured at the object surface at the time moment $t$. Lyubimov et al. $[8,10,11]$ have shown that for $I_{0}-I \ll I_{0}$, when the perturbation theory may be used, the relative shadow can be expressed in the form of the fundamental equation of the PAT method for the case of the time-domain measurement technique as follows:

$$
g(L, t)=\int_{L} \frac{c}{n_{0} v(l)}\left(\int_{V} S\left(\mathbf{r}_{1}, \tau\right) P\left(\mathbf{r}_{1}, \tau ; \mathbf{r}, t\right) d^{3} r_{1}\right) d l,
$$

where $c$ is a light velocity in vacuum, $n_{0}$ is a refraction index of a homogeneous media, $L$ is a full PAT from a source to a receiver, $l$ is a path traversed by the mass center of the photon distribution along a PAT over the time $\tau, v(l)$ is a velocity of the mass center as a function of path $l$, and $S(\mathbf{r}, t)$ is an inhomogeneity distribution function. In the general case, function $S(\mathbf{r}, t)$ describes local disturbances $\delta D(\mathbf{r}), \delta \mu_{a}(\mathbf{r})$, and $\delta n(\mathbf{r})$ of the diffusion coefficient $D(\mathbf{r})$, the absorption coefficient $\mu_{a}(\mathbf{r})$, and the refraction index $n(\mathbf{r})$, correspondingly, and is defined by the expression $[4,5]$

$$
\begin{aligned}
S(\mathbf{r}, t)= & \frac{\mu_{a 0} \delta D(\mathbf{r})}{D_{0}}-\delta \mu_{a}(\mathbf{r}) \\
& +\left(\frac{n_{0} \delta D(\mathbf{r})}{c D_{0}}-\frac{\delta n(\mathbf{r})}{c}\right) \frac{\partial}{\partial t} \ln \varphi_{0}(\mathbf{r}, t) .
\end{aligned}
$$

Here, the subscript " 0 " corresponds to a homogeneous media. The principal possibility to separate the distributions of optical parameters is substantiated in [16]. It is based on a simplification of (6) and shadow measurements for different 
values of time-gating delay $t$. In the present paper, without loss of generality, we consider a practically important case of absorbing inhomogeneity given by the absorption coefficient $\mu_{a}(\mathbf{r})=\mu_{a 0}+\delta \mu_{a}(\mathbf{r})$, when $\delta D(\mathbf{r})=0$ and $\delta n(\mathbf{r})=0$.

\subsection{Implementation of backprojection algorithm}

Using the approaches of projection tomography, the fundamental equation of the PAT method may be directly inverted in relation to the function

$$
\langle S(\mathbf{r}, t)\rangle=\int_{V} S\left(\mathbf{r}_{1}, \tau\right) P\left(\mathbf{r}_{1}, \tau ; \mathbf{r}, t\right) d^{3} r_{1},
$$

namely, the function blurred due to averaging over the spatial distribution of photons, which form the signal measured by the receiver at the moment $t$. The FBP implementation used by us for reconstruction of function (7) is based on a simple approximation of a curvilinear PAT and a velocity of the mass center of the photon distribution. In $[8,9]$, Lyubimov et al. have shown that for most object geometries, wherein a source and a receiver, lie on the boundary of the object, a three-segment polygonal line can be used to approximate a curvilinear PAT. The first and the end segments of this broken line are normal to the object boundary and equal in length, and the middle segment connects their ends.

The velocity of the photon distribution mass center is inversely proportional to the distance from the object boundary when moving the center along the outer segments of the broken PAT, and takes the stationary value when moving along the middle segment. Let us consider a common 2D geometry for DOT, when sources and receivers lie around the boundary of a circular object at equal step angles. Our investigations [11-15] show that by choosing the optimal values of the time-gating delay of receivers, the length of the middle segment of the broken PAT may be greatly longer than the first and the end ones. Moreover, the time-gating delays for different source-receiver pairs can be chosen so that the lengths of the outer segments for all broken-line approximations of the PATs are equal. Thus, we can put an extensive internal region of the object, corresponding to the middle segments of the broken PATs. Such region is denoted in Figure 1 by an internal circle. For geometry chosen, each PAT is defined in the space by the angular locations $\gamma_{s}$ and $\gamma_{d}$ of source $S$ and receiver $D$, correspondingly (Figure 1 ). As initial conditions for the inverse problem, the relative shadows $g\left(\gamma_{s}, \gamma_{d}\right)$ induced by inhomogeneities are known for each source-receiver pair. Let the inhomogeneities be localized in the region corresponding to the middle segments of the broken PATs. In this case the measurement results $g\left(\gamma_{s}, \gamma_{d}\right)$ in the first-order approximation can be defined by line integrals along the middle segments of the PATs (Figure 1). The relative shadows $g\left(\gamma_{s}, \gamma_{d}\right)$ may be approximately considered as the fan beam projections of straight rays transmitted from point sources to point receivers, each extrapolated to the internal circle (Figure 1). As it is clear from (6), in the case of absorbing inhomogeneity, the function $S(\mathbf{r}, t)$ can be defined as

$$
S(\mathbf{r}, t)=-\delta \mu_{a}(\mathbf{r})
$$

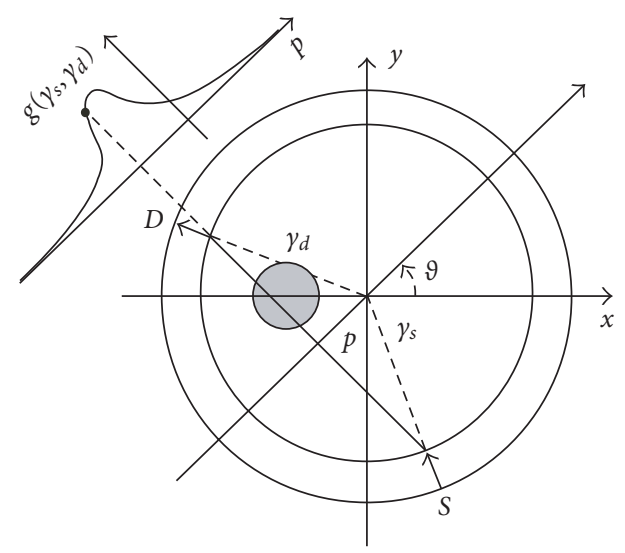

FIgURE 1: The geometry of the image reconstruction problem.

Taking into account that inside the object the velocity $v(l)$ is approximated by a constant, we can modify the fan beam projection data $g\left(\gamma_{s}, \gamma_{d}\right)$ so that only the function $\left\langle\delta \mu_{a}(\mathbf{r})\right\rangle$ remains under integral sign in (5). Thus, in the case of the absorbing inhomogeneity, the inverse problem of DOT reduces to solution of the following integral equation:

$$
g^{\prime}\left(\gamma_{s}, \gamma_{d}\right)=\int_{L^{a v}}\left\langle\delta \mu_{a}(\mathbf{r})\right\rangle d l
$$

where $g^{\prime}\left(\gamma_{s}, \gamma_{d}\right)$ is the modified projection data $g\left(\gamma_{s}, \gamma_{d}\right), L^{a v}$ is the middle segment of the broken approximation of $L$. Equation (9) is a full analogue of the Radon transform and may be solved by using FBP with standard convolution filtration. We implement it using the sequence of two steps as follows.

(1) Convert the fan beam projections $g^{\prime}\left(\gamma_{s}, \gamma_{d}\right)$ to the parallel ones $\hat{g}(p, \vartheta)$ by a 2D spline interpolation [24]. The first argument $p$ of function $\hat{g}(p, \vartheta)$ denotes a count along the parallel projection, and the second one $\vartheta$ is an angular aspect for which this projection is registered (Figure 1).

(2) Apply the standard FBP realization for the parallel beam geometry with filtration in frequency domain $[25]$ to the converted projections $\hat{g}(p, \vartheta)$.

We develop the Matlab code, wherein steps 1 and 2 are realized with the use of the basic functions "griddata $(\cdot)$ " and "iradon $(\cdot)$, , correspondingly [26]. The detailed description of the algorithm implemented is given in [14] and is not a main subject of this paper.

\section{POST-RECONSTRUCTION RESTORATION OF TOMOGRAMS}

\subsection{Validation of linear spatially variant blur model}

The PSF of a visualization system is defined as the image of an infinitesimally small point object and specifies how points in the image are distorted. But the PSF may be used for system description if a model of a linear filter [27] is available. Such a model is ordinarily used in traditional 
medical tomography (X-ray computed tomography, magnetic resonance intrascopy, single-photon emission tomography, positron emission tomography, etc.). A diffuse optical tomograph in general is not a linear filter because of the absence of regular rectilinear trajectories of the photons. However, the PAT method with the FBP realization has the following features.

(1) Our concept proposes the conditional PATs to be used for reconstruction as regular trajectories.

(2) The object region corresponding to the rectilinear parts of the PATs is only reconstructed.

(3) The reconstruction algorithm, all of whose operations and transformations are linear, is used.

These features in our opinion warrant the application of a model of a linear filter in given particular case of DOT. Therefore, the PSF may be assumed for describing the blur due to reconstruction.

Let us consider at once the variance of the PSF against spatial shift. The time integral of function $P\left(\mathbf{r}_{1}, \tau ; \mathbf{r}, t\right)$ for each $\tau$ describes instantaneous distribution of diffuse photon trajectories. At time moment $\tau=t$, this distribution forms a "banana-shaped" zone $[8,11,28]$ of the most probable trajectories of photons migrated from $(0,0)$ to $(\mathbf{r}, t)$. The effective width of this zone estimates the theoretical spatial resolution and is described by the standard root-mean-square deviation (RMSD) of photon position from the PAT as follows:

$$
\Delta(\mathbf{r}, t, \tau)=\left[\int_{V}\left|\mathbf{r}_{1}-\mathbf{R}\left(\mathbf{r}_{1}, t, \tau\right)\right|^{2} P\left(\mathbf{r}_{1}, \tau ; \mathbf{r}, t\right) d^{3} r_{1}\right]^{1 / 2} .
$$

In [8], Lyubimov has shown that RMSD depends slightly upon the object form and coincides virtually with that in the infinite media. Therefore, to estimate the resolution for the objects of complex forms, the simple formulas for the infinite media may be used. It is not difficult to show that in the case of the homogeneous infinite media, equations (2) and (10) are written as follows:

$$
P\left(\mathbf{r}_{1}, \tau ; \mathbf{r}, t\right)=(\sqrt{2 \pi} \sigma)^{-3} \exp \left(-\frac{\left|\mathbf{r} \tau / t-\mathbf{r}_{1}\right|^{2}}{2 \sigma^{2}}\right),
$$

where

$$
\begin{gathered}
\sigma=\sqrt{\frac{2 D_{0} c \tau}{n_{0}}\left(1-\frac{\tau}{t}\right)}, \\
\Delta(\mathbf{r}, t, \tau)=\sqrt{12 D_{0} c\left(t-\frac{|\mathbf{r}| n_{0}}{c}\right) \frac{\tau(t-\tau)}{n_{0} t^{2}}} .
\end{gathered}
$$

It is seen from $(11)$ that $P\left(\mathbf{r}_{1}, \tau ; \mathbf{r}, t\right)$ is not a function of the difference $\mathbf{r}-\mathbf{r}_{1}$ even for the simplest geometry of the infinite space. Therefore, averaging (7) cannot be described by a convolution and the blur due to PAT reconstruction is spatially variant. Numerically, the spatial variance can be estimated by (12). For example, let us have a circular scattering object with diameter $d=6.8 \mathrm{~cm}$ and optical parameters $D_{0}=0.066 \mathrm{~cm}$ and $n_{0}=1.4$. Let us assume a source and a receiver to lie on the object boundary and to be poles asunder. Then the calculation under (12) for time-gating delay $t=600 \mathrm{ps}$ gives the following results:

$$
\left.\Delta\right|_{\tau=t / 2} \approx 1 \mathrm{~cm},\left.\quad \Delta\right|_{\tau=t / 4}=\left.\Delta\right|_{\tau=3 t / 4} \approx 0.87 \mathrm{~cm} .
$$

The first value obtained estimates the spatial resolution for the central region of the object and the second one estimates the resolution for regions remote from the center over a half radius. According to (12), as the object boundary is approached, the theoretical resolution tends to zero. Thus, the resolution and, therefore, the PSF describing the blur are strongly variant against spatial shift. It means that the spatially variant blur model may be exclusively assumed for restoration of the PAT tomograms.

A generic spatially variant blur would require a point source at every pixel location to fully describe the blurring operation. Since it is not possible to do this, even for small images, some approximations should be done. There are several approaches to restoration of images degraded by spatially variant blur. One of them is based on a geometrical coordinate transformation [29-31] and uses coordinate distortions or known symmetries to transform the spatially variant PSF into one that is spatially invariant. After applying a spatially invariant restoration method, inverse coordinate distortion is used to obtain the result. This approach does not suit for us since the coordinate transformation functions need to be known explicitly. Another approach considered, for example, in [32-34], is based on the assumption that the blur is approximately spatially invariant in small subregions of the image domain. Each subregion is restored using its own spatially invariant PSF, and the results are then sewn together to obtain the restored image. This approach is laborious and, moreover, gives the blocking artifacts at the subregion boundaries. To restore the PAT images, we assume the blur model recently developed by Nagy et al. [17-19]. According to it the blurred image is partitioned into subregions with the spatially invariant PSFs. However, rather than deblurring the individual subregions locally and then sewing the individual results together, this method interpolates the individual PSFs, and restores the image globally. It is clear that the accuracy of such method depends on the number of subregions into which the image domain is partitioned. The partitioning where the size of one subregion tends to a spatial resolution seems to be sufficient for obtaining a restoration result of good quality.

\subsection{Implementation of blur model}

Let $\mathbf{x}$ be a vector representing the unknown true image of an absorbing inhomogeneity $\delta \mu_{a}(\mathbf{r})$ and let $\mathbf{b}$ be a vector representing the reconstructed image $\left\langle\delta \mu_{a}(\mathbf{r})\right\rangle$ blurred due to averaging (7). The spatially variant blur model of Nagy et al. is described by a system of linear algebraic equations

$$
\mathbf{b}=\mathbf{A} \cdot \mathbf{x}
$$

where $\mathbf{A}$ is a large ill-conditioned matrix that models the blurring operator (blurring matrix). If the image is partitioned into $m$ subregions, the matrix $\mathbf{A}$ has the following 
structure:

$$
\mathbf{A}=\sum_{j=1}^{m} \mathbf{D}_{j} \mathbf{A}_{j}
$$

where $\mathbf{A}_{j}$ are the banded block Toeplitz matrices with banded Toeplitz blocks $[18,35]$ and $\mathbf{D}_{j}$ are diagonal matrices satisfying the condition

$$
\sum_{j=1}^{m} \mathbf{D}_{j}=\mathbf{I}
$$

where I is the identity matrix. The piecewise constant interpolation implemented implies that the $i$ th diagonal entry of $\mathbf{D}_{j}$ is one if the $i$ th pixel is in the $j$ th subregion, and zero otherwise. Each matrix $\mathbf{A}_{j}$ is uniquely determined by a single column $\mathbf{a}_{j}$ that contains all of the nonzero values in $\mathbf{A}_{j}$. This vector $\mathbf{a}_{j}$ is obtained from an invariant PSF corresponding to the $j$ th subregion $\left(\mathbf{P S F}_{j}\right)$ as follows:

$$
\mathbf{a}_{j}=\operatorname{vec}\left(\mathbf{P S F}_{j}^{T}\right)
$$

where the operator "vec $(\cdot)$ " transforms matrices into vectors by stacking the columns. The "banding" of matrix $\mathbf{A}_{j}$ means that the matrix-vector multiplication product $\mathbf{D}_{j} \mathbf{A}_{j} \mathbf{z}$, where $\mathbf{z}$ is any vector defined into the image domain and depends on the values of $\mathbf{z}$ in the $j$ th subregion, as well as on values in other subregions within a width of the borders of the $j$ th subregion. The matrix-vector multiplication procedure is implemented in Nagy's Matlab package "Restore Tools" [36] by means of the $2 \mathrm{D}$ discrete fast Fourier transform and is fully described in [19].

To simulate the invariant PSF corresponding to individual subregion, first of all we must choose a characteristic point and specify a point inhomogeneity in it. It is advisable to choose the center of subregion for location of the point inhomogeneity. The algorithm of individual PSF simulation includes two steps as follows.

(1) Simulate the relative shadows caused by the point inhomogeneity.

(2) Reconstruct the PSF from simulated shadows by the PAT method with the FBP realization.

The relative shadows caused by the point inhomogeneity are simulated via the numerical solution of the time-dependent diffusion equation with the use of the finite element method (FEM). To guarantee against inaccuracy of calculations, we optimize the finite element mesh so that it is strongly compressed in the vicinity of the point inhomogeneity location. Thereto the Matlab function "adaptmesh $(\cdot)$ " is used. For FEM calculations, the point inhomogeneity is assigned by three equal values into the nodes of the little triangle on the center of compressed vicinity. The example of the optimized mesh is given in Figure 2(a). To reconstruct the PSF from simulated shadows, the backprojection algorithm implemented as described in Section 2 is used. The example of the reconstruction result corresponding to the mesh of Figure 2(a) is presented as surface plot in Figure 2(b).

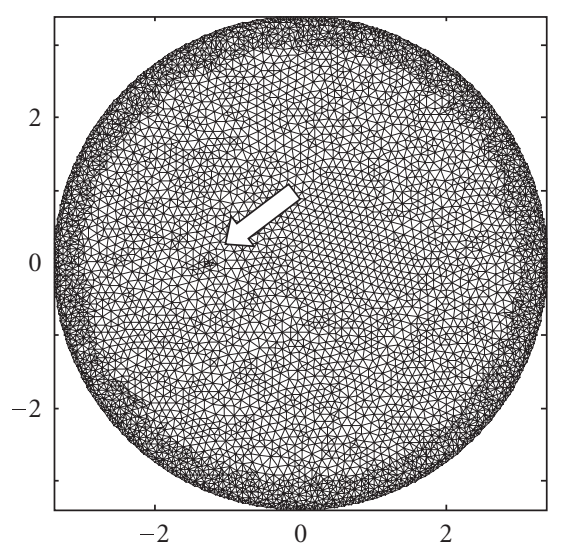

(a)

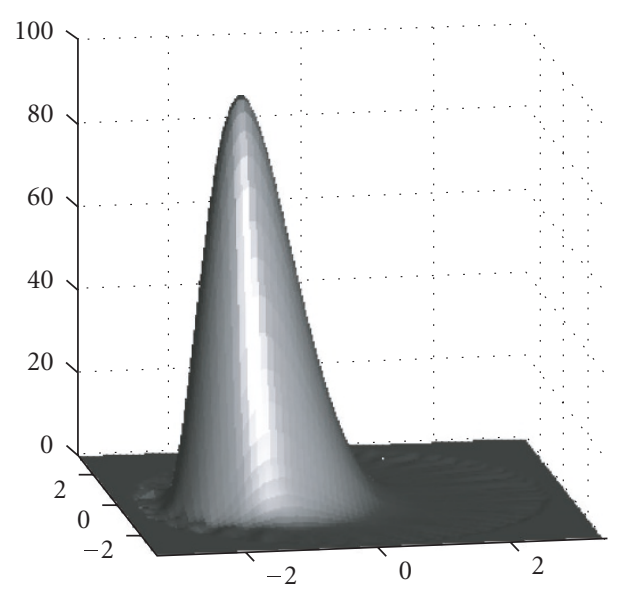

(b)

FIGURE 2: Simulation of the individual PSF: (a) high-resolution finite element mesh with the compressed vicinity, (b) the simulation result corresponding to the mesh.

It is clear that some laborious numerical calculations for various locations of the point inhomogeneity should be made. To simplify the problem in the case of circular geometry, it is desirable to consider polar coordinates $(p, \vartheta)$. It is easy to see that the PSF for a constant radial distance $p$ has the same shape for all angular positions $\vartheta$ but is rotated through angle $\vartheta$. In other words, the PSF is spatially invariant with respect to the angular position. Therefore, the PSFs need to be calculated only for different values of $p$ at an angle $0^{\circ}$. At any other angle, the PSFs can be rotated, in real time, using a bilinear interpolation. The array of the invariant PSFs calculated for the case of image partitioning into $5 \times 5$ subregions is presented in Figure 3.

\subsection{Restoration algorithms}

After constructing the blurring matrix A, an acceptable algorithm should be chosen to solve system (14) for unknown vector $\mathbf{x}$. Because of the large dimensions of the linear 


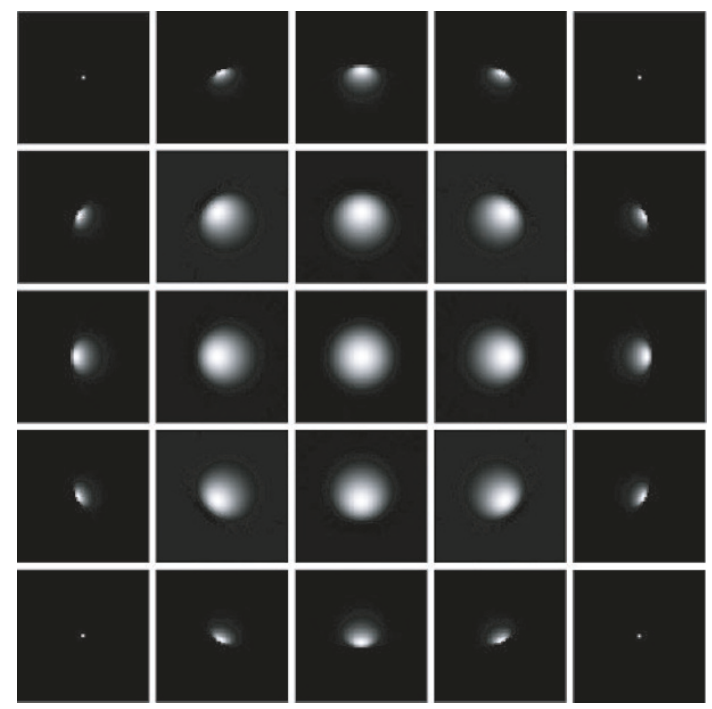

Figure 3: The $5 \times 5$ array of the invariant PSFs corresponding to individual subregions.

system, iterative algorithms are typically used to compute approximations of $\mathbf{x}$.

Those include a variety of conjugate gradient-type algorithms $[20,37,38]$, the steepest descent algorithms [21, 22, $38,39]$, the expectation-maximization algorithms [40-42], and many others [43]. Since no one iterative algorithm is optimal for all image restoration problems, the study of iterative algorithms is an important area of research. In the present paper, we consider the conjugate gradient algorithm CGLS [20] and the steepest descent algorithm MRNSD [21, 22] implemented in Nagy's package by the functions "CGLS(.)" and “MRNSD(·)," correspondingly. These algorithms represent two different approaches: a Krylov subspace method applied to the normal equations and a simple descent scheme with enforcing a nonnegativity constraint on solution. The step sequences describing the algorithms are given in Figure 4. The operator $\|\cdot\|$ denotes an Euclidian norm, the function "diag $(\cdot)$ " produces the diagonal matrix containing the initial vector.

Both CGLS and MRNSD are easy to implement and converge more faster than, for example, the expectationmaximization algorithms $[22,44]$. Both algorithms exhibit a semi-convergence behavior [38] with respect to the relative error $\left\|\mathbf{x}_{k}-\mathbf{x}\right\| /\|\mathbf{x}\|$, where $\mathbf{x}_{k}$ is the approximation of $\mathbf{x}$ at the $k$ th iteration. It means that, as the iterative process goes on, the relative error begins to decrease and, after some optimal iteration, begins to rise. By stopping the iteration when the error is low, we obtain a good regularized approximation of the solution. Thus, the iteration number plays the role of the regularization parameter. This is very important for us, as the matrix $\mathbf{A}$ is severely ill-conditioned and regularization must be necessarily incorporated. To estimate the optimal iteration number, we use the following blurring residual [45] that measures the image quality change after beginning the restoration process:

$$
\beta_{k}=\frac{\left\|\mathbf{x}_{k}-\mathbf{x}\right\|}{\|\mathbf{b}-\mathbf{x}\|} \%
$$

Like the relative error, the blurring residual has a minimum that corresponds to the optimal iteration number. Note that we do not know the true image (vector $\mathbf{x}$ ) in clinical applications of DOT. However, using criterion $\beta_{k} \rightarrow \mathrm{min}$, it is possible to calibrate the algorithms on relation to the optimal iteration number via experiments (including numerical experiments) with phantoms. In general many different practical cases of optical inhomogeneities can be considered for calibration. In clinical explorations, the particular case is chosen from a priori information, which the blurred tomograms contain after reconstruction. Further, regularization can be enforced in a variety of other ways, including Tikhonov [46], iteration truncation [37, 47], as well as mixed approaches [48]. Preconditioned iterative regularization by truncating the iterations is an effective approach to accelerate the rate of convergence. Such preconditioning is implemented in Nagy's package for both algorithms (CGLS and MRNSD) considered. In general, preconditioning amounts to find a nonsingular matrix $\mathbf{C}$, such that $\mathbf{C} \approx \mathbf{A}$ and such that $\mathbf{C}$ can be easily inverted. The iterative method is then applied to preconditioned system

$$
\mathbf{C}^{-1} \mathbf{b}=\mathbf{C}^{-1} \mathbf{A} \cdot \mathbf{x} \text {. }
$$

The appearance of matrix $\mathbf{C}$ is defined by the regularization parameter $\lambda<1$ that characterizes a step size at each iteration. In this paper, we consider two methods for calculating $\lambda$ : generalized cross validation (GCV) method [47] and method based on criterion of blurring residual minimum. In the first case we assume that a solution computed on a reduced set of data points should give a good estimate of missing points. The GCV method finds a function of $\lambda$ that measures the errors in these estimates. The minimum of this GCV function corresponds to the optimal regularization parameter. In the second case we calculate blurring residual (18) for different numbers of iterations and different discrete values of $\lambda$, taken with the step $\Delta \lambda$. The minimum of blurring residual corresponds to optimal number of iterations and the optimal regularization parameter.

The main reason of choosing MRNSD for PAT image restoration is that this algorithm enforces a nonnegativity constraint on the solution approximation at each iteration. Such enforcing produces much more accurate approximate solutions in many practical cases of nonnegative true image $[21,22,49]$. In DOT (e.g., optical mammography), when a tumor structure is detected, one can expect that the disturbances of optical parameters are not random heterogeneous distributions, but they are smooth nonnegative functions standing out against a zero-mean background and forming the macroinhomogeneity images. Indeed, the typical values of absorption coefficient lie within the range between 0.04 and $0.06 \mathrm{~cm}^{-1}$ for healthy breast tissue, and between 0.06 and $0.1 \mathrm{~cm}^{-1}$ for breast tumor $[50,51]$. Thus, we have the nonnegative true image $\delta \mu_{a}(\mathbf{r})$. This a priori knowledge gives the 


\begin{tabular}{|ll|}
\hline \multicolumn{1}{|c|}{ CGLS } & \\
$\mathbf{x}=\mathbf{b}$ & $\mathbf{x}=\mathbf{b}$ \\
$\mathbf{r}=\mathbf{b}-\mathbf{A} \mathbf{x}$ & $\mathbf{g}=\mathbf{A}^{T}(\mathbf{A x}-\mathbf{b})$ \\
$\mathbf{g}=\mathbf{A}^{T} \mathbf{r}$ & $\mathbf{X}=\operatorname{diag}(\mathbf{x})$ \\
$\gamma=\|\mathbf{g}\|^{2}$ & $\gamma=\mathbf{g}^{T} \mathbf{X g}$ \\
for $k=1,2, \ldots$ & for $k=1,2, \ldots$ \\
$\quad$ if $k=1, \mathbf{s}=\mathbf{g}$ & $\mathbf{s}=-\mathbf{X g}$ \\
otherwise $\mathbf{s}=\mathbf{g}+\left(\gamma / \gamma_{\text {old }}\right) \mathbf{s}$ & $\mathbf{u}=\mathbf{A s}$ \\
$\mathbf{u}=\mathbf{A s}$ & $\alpha=\min \left(\gamma / \mathbf{u}^{T} \mathbf{u}, \min _{s_{i}<0}\left(-x_{i} / s_{i}\right)\right)$ \\
$\alpha=\gamma /\|\mathbf{u}\|^{2}$ & $\mathbf{x}=\mathbf{x}+\alpha \mathbf{s}$ \\
$\quad \mathbf{x}=\mathbf{x}+\alpha \mathbf{s}$ & $\mathbf{X}=\operatorname{diag}(\mathbf{x})$ \\
$\mathbf{r}=\mathbf{r}-\alpha \mathbf{u}$ & $\mathbf{z}=\mathbf{A}^{T} \mathbf{u}$ \\
$\mathbf{g}=\mathbf{A}^{T} \mathbf{r}$ & $\mathbf{g}=\mathbf{g}+\alpha \mathbf{z}$ \\
$\gamma_{\text {old }}=\gamma, \gamma=\|\mathbf{g}\|^{2}$ & $\gamma=\mathbf{g}^{T} \mathbf{X g}$ \\
end & end \\
\hline
\end{tabular}

FIgURE 4: The step sequences describing the restoration algorithms.

right to apply constrained MRNSD and change negative values for zeros after applying unconstrained CGLS.

\section{RESULTS AND ANALYSIS}

To demonstrate the effect of improving the spatial resolution of the PAT tomograms, a numerical experiment was conducted, wherein circular strongly scattering objects were reconstructed and then restored. The diameter of objects was equal to $6.8 \mathrm{~cm}$. The refraction index, coefficients of diffusion and absorption of the objects were equal to $1.4,0.066 \mathrm{~cm}$, and $0.05 \mathrm{~cm}^{-1}$, correspondingly. We considered two sets of phantoms. Each phantom of the first set contained a circular absorbing inhomogeneity with the diameter equal to $1 \mathrm{~cm}(\mathrm{ab}-$ sorption coefficient was equal to $0.075 \mathrm{~cm}^{-1}$ ). In one of the cases, the inhomogeneity was located in the center of the object, in the two others it was displaced from the center by 1.25 and $2.5 \mathrm{~cm}$, correspondingly. The second set of phantoms was designated to measure the modulation transfer function (MTF) that was used by us for rough estimation of the spatial resolution limit. We used five circular strongly scattering objects, each containing two circular absorbing inhomogeneities equal in diameter. The diameter and optical parameters of these objects, as well as the absorption coefficient of inhomogeneities, were identical to those of the phantoms of the first set. The distance between inhomogeneities was equal to their diameter. Diameters of inhomogeneities of different objects were equal to $1.4,1.2,1.0,0.8$, and $0.6 \mathrm{~cm}$. Sources (32) and receivers (32) were installed along the perimeter of the objects at equal step angles $\left(11.25^{\circ}\right)$; the angular distance between the nearest-neighbor source and receiver constituted $5.625^{\circ}$. The relative shadows caused by the absorbing inhomogeneity were simulated via the numerical solution of the time-dependent diffusion equation for the instantaneous point source with the use of the FEM method. The time-gating delays of the receivers were chosen so that the lengths of the outer segments for all broken-line approximations of PATs were equal to $0.3 \mathrm{~cm}$. Thus, the internal region of objects, corresponding to the middle segments of broken lines, was equal to $6.2 \mathrm{~cm}$ in diameter. Reconstruction of each phantom (its internal region) with the use of FBP was realized onto rectangular grid $63 * 63$. Under visualization the boundary region corresponding to outer segments of broken PATs was filled by zeros and full image domain was shown in each case.

The reconstruction results for phantoms of the first set are presented in Figure 5 as gray-level images in comparison with the best results of deblurring. The blurred images are given on the left. The central column of images corresponds to the restoration results obtained with the use of unpreconditioned CGLS. And the images restored by unpreconditioned MRNSD are presented in Figure 5 on the right. The upper images correspond to the object with the central inhomogeneity, the central row of images - to the object with the inhomogeneity displaced from the center by $1.25 \mathrm{~cm}$ and the bottom ones - to the object with the inhomogeneity displaced by $2.5 \mathrm{~cm}$. White points in the images show the object boundaries known a priori. The coordinate axes are graduated in centimeters and the intensity scalein reverse centimeters. The blurred reconstructions and the results of their restoration with the use of unpreconditioned algorithms for phantoms of the second set are given as surface plots in Figure 6. Like in Figure 5, the blurred images are given on the left. The CGLS restorations are shown in the center, and the MRNSD ones-on the right of Figure 6. The sequence of image triplets from top to bottom corresponds to a scale of inhomogeneity diameters from 1.4 to $0.6 \mathrm{~cm}$. The intensity values are separately normalized for each image and shown on a percent scale (vertical axes of the plots). The restoration results presented in Figures 5 and 6 correspond to the optimal iteration number and the image partitioning into $5 \times 5$ subregions. The optimal iteration number obtained by the criterion of blurring residual minimum is equal to 15 in the case of unpreconditioned CGLS and to 9 in the case of unpreconditioned MRNSD, respectively. The number of subregions into which the image domain is partitioned $(5 \times 5)$ was chosen starting from compromise between the restoration quality and the restoration time. Table 1 shows how the restoration time per iteration grows as the number of image subregions increases. From Table 1 it follows that the image 

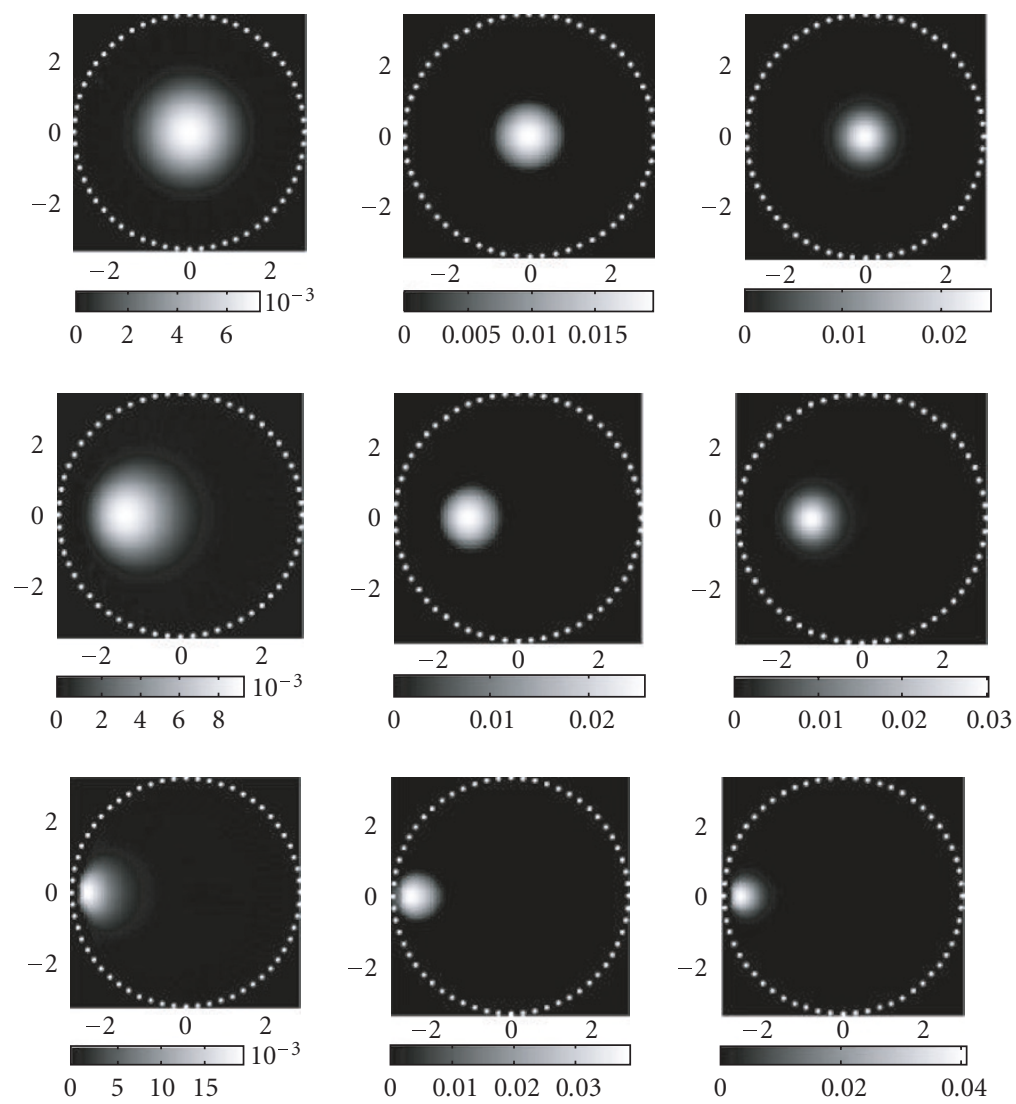

FIGURE 5: The best results of restoration by unpreconditioned algorithms in comparison with the results of blurred image reconstruction: the first set of phantoms.

TABLE 1: The restoration time per iteration depending on the number of image subregions. The computation time is given in seconds for an Intel PC with 1.7 GHz Pentium 4 processor and 256-MB RAM.

\begin{tabular}{lccc}
\hline & $1 \times 1$ & $3 \times 3$ & $5 \times 5$ \\
\hline CGLS & 0.1 & 0.8 & 2.1 \\
MRNSD & 0.1 & 0.9 & 2.3 \\
\hline
\end{tabular}

partitioning into more than $5 \times 5$ subregions cannot satisfy demand of real-time medical explorations.

The bottom images of Figure 5 show that, as the object boundary is approached, the restoration quality becomes slightly worse. That is why the backprojection algorithm does not correctly reconstruct the boundary region of an object. When the inhomogeneities are remote well away from the boundary, both unpreconditioned algorithms restore the tomograms without visible distortions and give a good gain in resolution, which is numerically estimated by MTF, as it is described below.

Figure 7 presents the restoration results obtained with the use of preconditioned MRNSD. The left column of images corresponds to the regularization parameter calculated by GCV method $(\lambda=0.003)$. To obtain the central restora- tions, we used preconditioner with $\lambda=0.1$. This value of the regularization parameter was found by the criterion of blurring residual minimum. The right column of images in Figure 7 shows the result of restoration by unpreconditioned MRNSD for comparison. As before the image domain was partitioned into $5 \times 5$ subregions. The optimal iteration number in the cases of preconditioned algorithm was equal to 3. Thus, preconditioners allow the restoration procedure to be accelerated. But, as it follows from Figure 7, preconditioned algorithm distort the form of inhomogeneities being restored. We can conjecture that the image partitioning into $5 \times 5$ subregions is not enough to obtain good quality of restoration by preconditioned algorithms. As we save computational time, the image partitioning number may be increased. Moreover, to restore a local region of inhomogeneity location, the PSFs can be simulated for each pixel of such region. Can we increase the restoration accuracy for preconditioned algorithms in this case? It is advisable to investigate this question in the future.

In view of ill-posed nature of the problem the restoration algorithms should be tested for noise immunity. In timedomain DOT, the random error of measurements is due to quantum noise. We incorporated noise with a standard deviation of 5,10 , and $20 \%$ of the maximum value into the relative shadows $g\left(\gamma_{s}, \gamma_{d}\right)$. Noisy sinograms (gray-level maps 

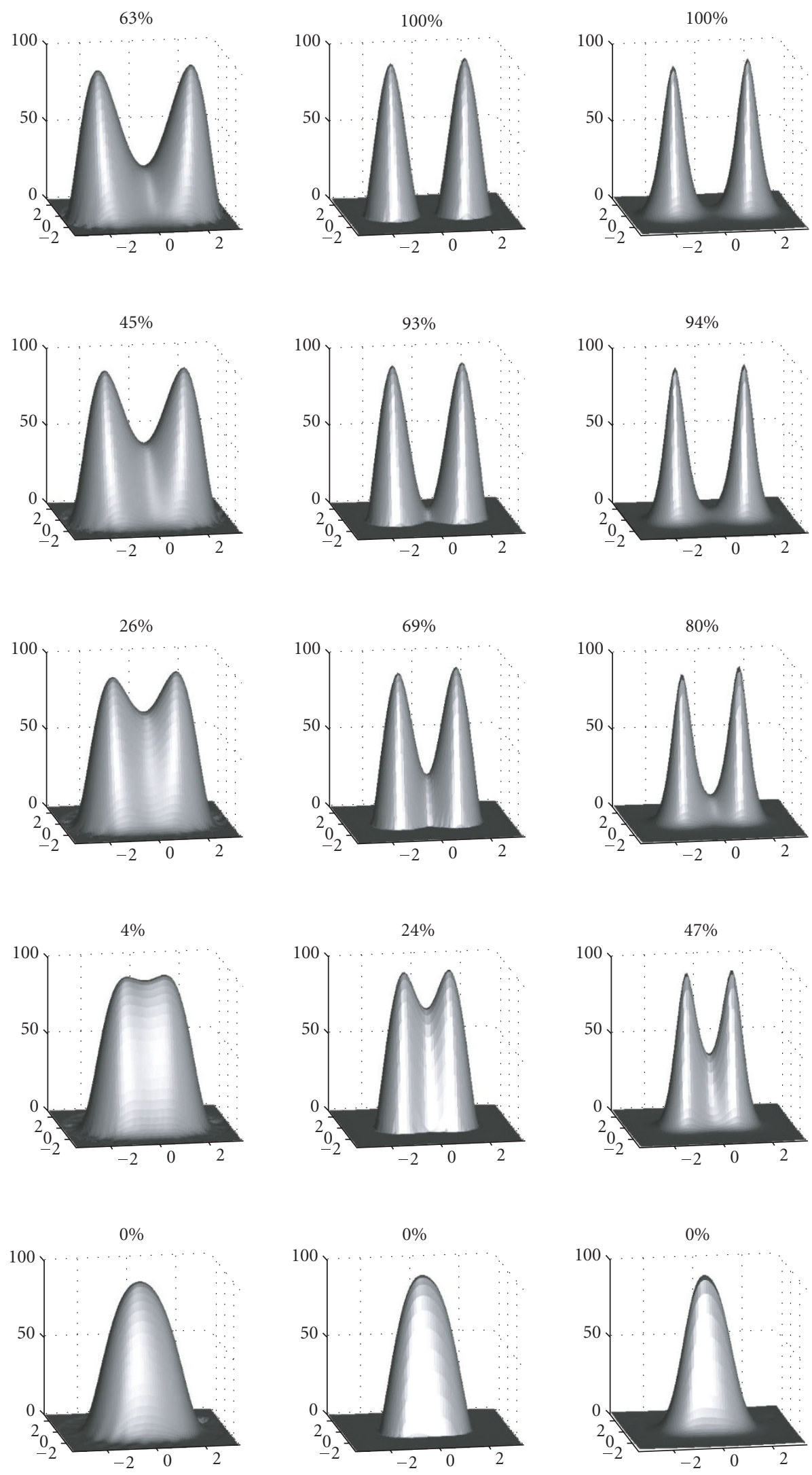

FIGURE 6: The best results of restoration by unpreconditioned algorithms in comparison with the results of blurred image reconstruction: the second set of phantoms. 

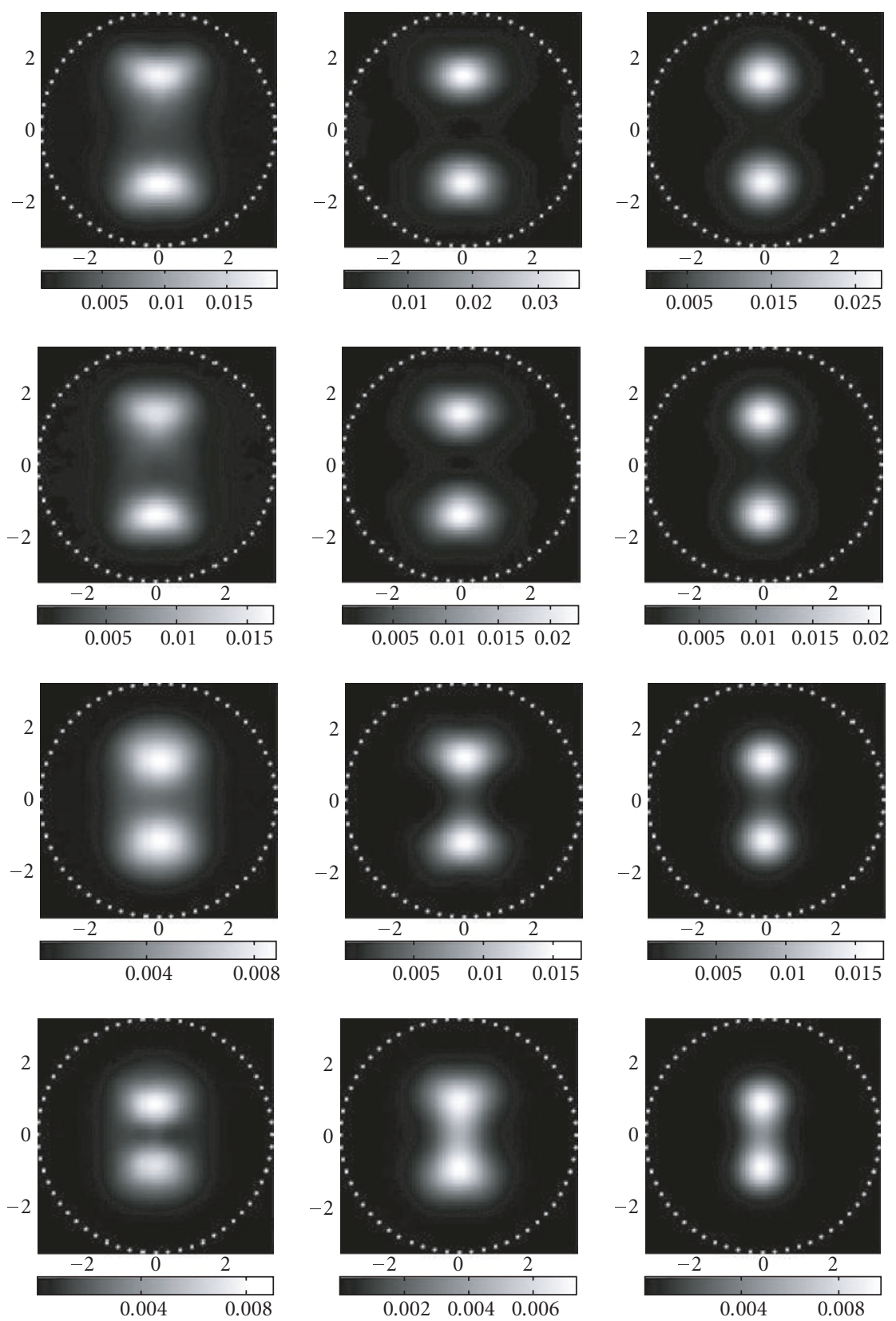

FIGURE 7: Comparison of the restoration results obtained with the use of preconditioned MRNSD (left and center) and unpreconditioned one (right): the second set of phantoms. Diameters of inhomogeneities from top to bottom are equal to $1.4,1.2,1.0$, and $0.8 \mathrm{~cm}$.

of shadow distributions over the index ranges of the source and the receiver) simulated for phantom with two inhomogeneities of diameter $1.4 \mathrm{~cm}$ are presented in Figure 8 on the left. The sinogram abscissa is the receiver index and the sinogram ordinate is the source index. The intensity scale is graduated in relative units. The central column of images shows the corresponding blurred tomograms reconstructed by FBP. The results of their restoration by unpreconditioned MRNSD are given on the right of Figure 8. You can see that there are distortions of the inhomogeneity forms in the cases of $10 \%$ - and $20 \%$-level noise. If the level of relative shadow noise is equal to $5 \%$, distortions are minimized (the right im- age in the top row). Unpreconditioned CGLS gives the similar results. Real quantum noise of time-resolved signal measurements depends on a number of photons in laser pulse and does not usually exceed the $2 \%$-level [52]. Thus, we can establish that unpreconditioned restoration algorithms are robust to measurement noise.

In conclusion it is interesting to compare the presented results with that obtained with a spatially invariant blur model. In the latter case, only one PSF calculated for point inhomogeneity in the center of image domain is used for restoration. Figure 9 shows the unpreconditioned MRNSD restorations of tomogram of phantom with two 

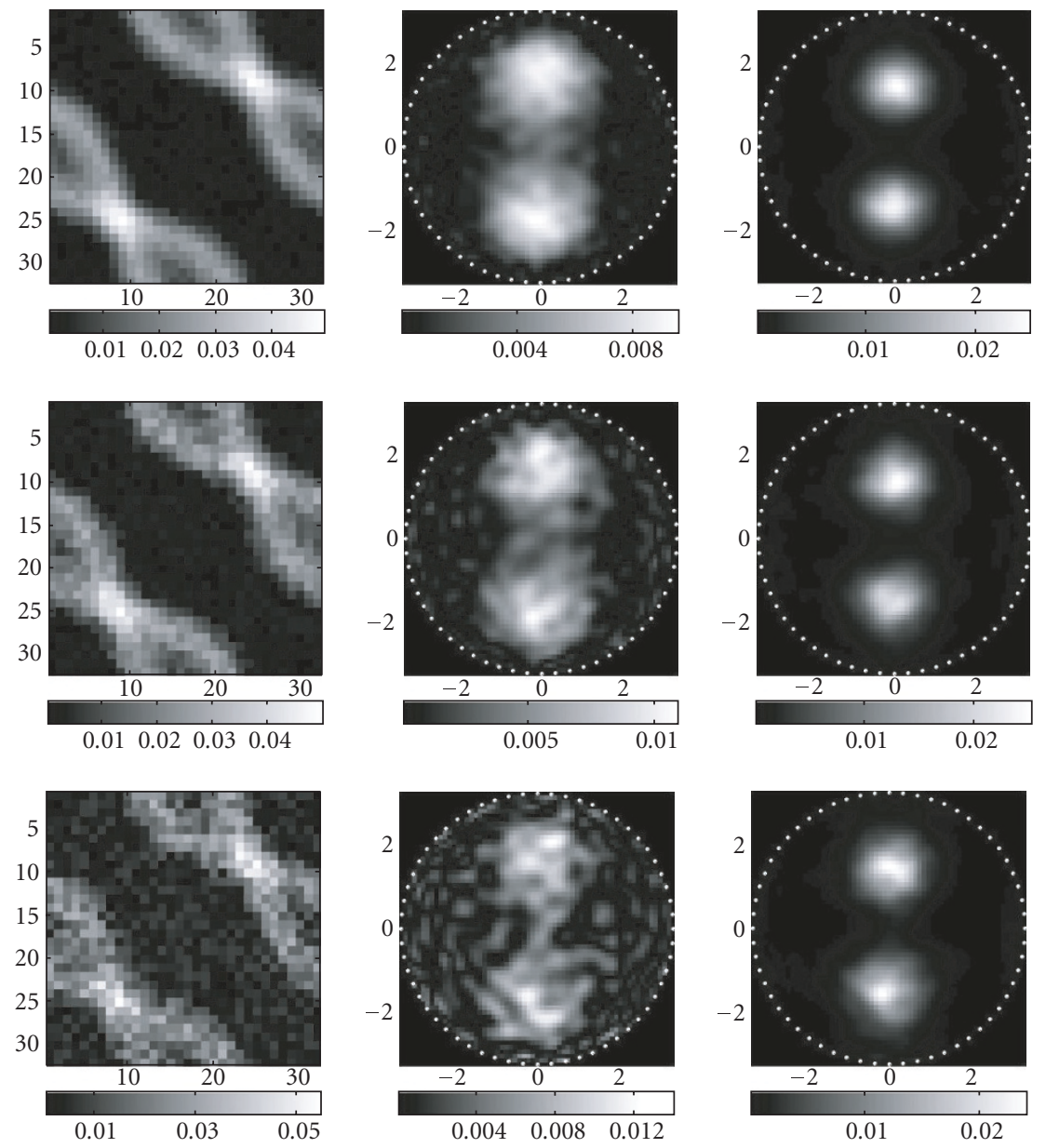

Figure 8: Noisy sinograms (left), blurred tomograms (center), and restorations by unpreconditioned MRNSD (right): phantom with two inhomogeneities of diameter $1.4 \mathrm{~cm}$. The noise level from top to bottom is equal to 5,10 , and $20 \%$.
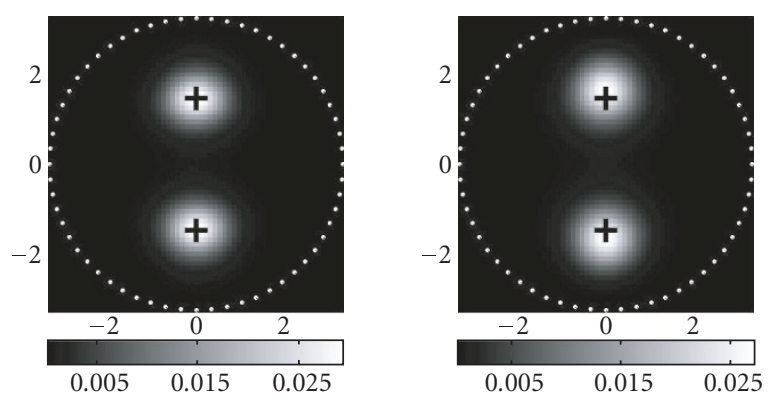

FIGURE 9: Comparison of the restoration results obtained with the spatially variant blur model (left) and with invariant one (right): phantom with two inhomogeneities of diameter $1.4 \mathrm{~cm}$.

inhomogeneities of diameter $1.4 \mathrm{~cm}$. The result obtained with the spatially variant model is given on the left of Figure 9. The right image represents the restoration with the use of the invariant PSF. The crosses in the figure mark the real positions of the centers of inhomogeneities. It is obvious that an application of the spatially invariant model leads to a shift of restored structures relative to their real locations. While, space-varying restoration gives the result without such distortions. This simple example justifies the theoretical assumption that the spatially variant blur model may be exclusively applied for restoration of the PAT tomograms.

On the base of the profile of each image of Figure 6, the modulation transfer coefficient (MTC) was determined as a relative depth of a dish between two peaks. The numerical results of MTC estimations are provided in Figure 6. Discrete values of spatial frequency were put in conformity with diameters of inhomogeneities. To estimate the MTF for the central region of the image domain, a dependence of the MTC on spatial frequency was accepted (Figure 10). It is seen from Figure 10 that with contrast of not less than $20 \%$, the spatial frequencies of no more than $0.54,0.67$, and 0.75 cycles/ $\mathrm{cm}$ can be reproduced on images blurred due to reconstruction and restored by CGLS and MRNSD, correspondingly. It means that the inhomogeneities with linear sizes of $0.92,0.75$, and $0.67 \mathrm{~cm}$ can be resolved on the coincident images. Thus, the gain in spatial resolution achieves $\sim 19 \%$ in the case of the CGLS restorations and $\sim 27 \%$ in the case of the MRNSD ones. The latter seems to be better 


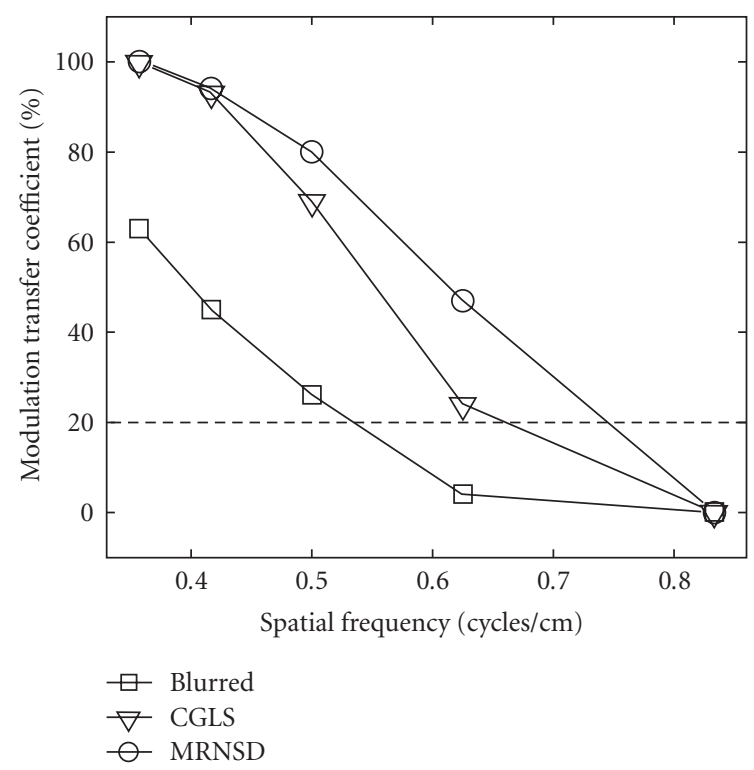

FIGURE 10: MTFs for blurred tomograms and restorations obtained with the use of unpreconditioned algorithms.

than the result obtained by using FBP with Vainberg filtration [12-15]. Therefore, to improve the resolution of the PAT reconstructions, not only special filtration of shadows but post-reconstruction restoration may be successfully used. Unlike the case of shadow filtration, the space-varying restoration model takes into account the blurring kernel and gives the results without any incline of the inhomogeneity profiles.

Our codes are in Matlab on a Windows XP Intel PC with 1.7-GHz Pentium 4 processor and 256-MB RAM. The FEM calculations of time-domain measurements on a highresolution mesh of 46,983 nodes and 10,326 elements take 4-5 min for one source. The time of reconstruction by our FBP realization for the measurement ratio $32 \times 32$ and the image grid $63 \times 63$ is less than $2 \mathrm{~s}$, including the projection transformation. If the image domain is partitioned into $5 \times 5$ subregions and unpreconditioned algorithms are used, the deblurring time is not more than $2.3 \mathrm{~s}$ per iteration (see Table 1). Thus, the reconstruction-restoration procedure takes approximately $30 \mathrm{~s}$ to produce satisfactory images. One can assume that the application of preconditioned algorithms for the spatially variant blur model with large partitioning number allows the full computational time to be saved. Moreover, in the future it is advisable to turn to a software medium faster than Matlab.

\section{CONCLUSION}

In this paper, we have examinated the possibility of the application of iterative restoration algorithms to improve the spatial resolution of diffuse optical tomograms reconstructed by the new photon average trajectories method. The spatially variant blur model was developed to restore images of inhomogeneities imbedded in a homogeneous strongly scattering object. To describe the blurring kernel, the spatially invariant point spread functions corresponding to different individual subregions of the image domain were simulated with the use of the finite element method. To study the efficiency of the blur model proposed, two iterative algorithms for solving a system of linear algebraic equations were implemented. Those are the conjugate gradient algorithm for least squares problem and the modified residual norm steepest descent algorithm. We have conducted a numerical experiment on reconstruction and restoration of circular phantoms with circular absorbing inhomogeneities. To reconstruct the blurred images under simulated time-domain measurements, the well-known filtered backprojection algorithm was used. The analysis of the restoration results presented shows that unpreconditioned algorithms are efficient for high-resolution deblurring the diffuse optical tomograms, in particular, when the inhomogeneity is remote well away from the boundary of the object. So, the steepest descent algorithm allows the $27 \%$-gain in spatial resolution to be obtained. The accuracy of space-varying restoration depends on the number of subregions into which the image domain is partitioned. The partitioning number $5 \times 5$ considered in this paper suits for unpreconditioned algorithms but seems to be not enough for effective use of accelerated algorithms with preconditioners. The further investigations of preconditioned algorithms should be carried on, as the application of preconditioners is available approach for saving computational time. More examples, in particular, for multitarget objects should be considered. The study of other restoration algorithms is also the subject of our short-term interest.

\section{ACKNOWLEDGMENTS}

The authors would like to thank Professor J. Nagy and his colleagues for their software package "Restore Tools" provided for the calculations. The authors also thank O. V. Lyamtsev, L. M. Yavorskaya, I. I. Kutuzov, O. V. Golubkina, G. B. Mordvinov, K. B. Domrachev, L. N. Soms, A. G. Murzin, and A. G. Kalintsev for fruitful and helpful discussions during preparation of the research. Konovalov and Vlasov were supported in part by the International Science and Technology Center under Grant 2151. Kravtsenyuk was supported in part by European Community, its 6th Community Frame and Program of Marie Curie Incoming International Fellowships MIF1-CT2005-008330 Real Time 3D Imaging.

\section{REFERENCES}

[1] S. R. Arridge, "Optical tomography in medical imaging," Inverse Problems, vol. 15, no. 2, pp. R41-R93, 1999.

[2] A. Yodh and B. Chance, "Spectroscopy and imaging with diffusing light," Physics Today, vol. 48, no. 3, pp. 34-40, 1995.

[3] A. H. Hielscher, A. D. Klose, and K. M. Hanson, "Gradientbased iterative image reconstruction scheme for time-resolved optical tomography," IEEE Transactions on Medical Imaging, vol. 18 , no. 3, pp. 262-271, 1999. 
[4] V. V. Lyubimov, "Physical foundations of the strongly scattering media laser tomography," in Laser Optics '95: Biomedical Applications of Lasers, vol. 2769 of Proceedings of SPIE, pp. 107-110, St. Petersburg, Russia, June 1996.

[5] V. V. Lyubimov, "Optical tomography of highly scattering media by using the first transmitted photons of ultrashort pulses," Optics and Spectroscopy, vol. 80, no. 4, pp. 616-619, 1996.

[6] V. V. Lyubimov, E. P. Mironov, A. G. Murzin, V. B. Volkonsky, and O. V. Kravtsenyuk, "Calculation of shadows induced by macroinhomogeneities located inside a strongly scattering object using the integration over the average photon path," in Photon Propagation in Tissues III, vol. 3194 of Proceedings of SPIE, pp. 409-416, San Remo, Italy, September 1997.

[7] V. V. Lyubimov, "On the spatial resolution of optical tomography of strongly scattering media with the use of the directly passing photons," Optics and Spectroscopy, vol. 86, no. 2, pp. 251-252, 1999.

[8] V. B. Volkonskii, O. V. Kravtsenyuk, V. V. Lyubimov, E. P. Mironov, and A. G. Murzin, "The use of statistical characteristics of photon trajectories for the tomographic studies of optical macroheterogeneities in strongly scattering objects," Optics and Spectroscopy, vol. 86, no. 2, pp. 253-260, 1999.

[9] O. V. Kravtsenyuk and V. V. Lyubimov, "Specific features of statistical characteristics of photon trajectories in a strongly scattering medium near an object surface," Optics and Spectroscopy, vol. 88, no. 4, pp. 608-614, 2000.

[10] O. V. Kravtsenyuk and V. V. Lyubimov, "Application of the method of smooth perturbations to the solution of problems of optical tomography of strongly scattering objects containing absorbing macroinhomogeneities," Optics and Spectroscopy, vol. 89, no. 1, pp. 107-112, 2000.

[11] V. V. Lyubimov, A. G. Kalintsev, A. B. Konovalov, et al., "Application of the photon average trajectories method to real-time reconstruction of tissue inhomogeneities in diffuse optical tomography of strongly scattering media," Physics in Medicine and Biology, vol. 47, no. 12, pp. 2109-2128, 2002.

[12] V. V. Lyubimov, A. B. Konovalov, I. I. Kutuzov, et al., "Influence of fast reconstruction algorithms on spatial resolution of optical diffuse tomography by photon average trajectories method," in Saratov Fall Meeting 2001: Optical Technologies in Biophysics and Medicine III, vol. 4707 of Proceedings of SPIE, pp. 53-59, Saratov, Russia, October 2002.

[13] A. B. Konovalov, V. V. Lyubimov, I. I. Kutuzov, et al., "Application of integral transform algorithms to high-resolution reconstruction of tissue inhomogeneities in medical diffuse optical tomography," in Optics in Health Care and Biomedical Optics: Diagnostics and Treatment, vol. 4916 of Proceedings of SPIE, pp. 9-21, Shanghai, China, October 2002.

[14] A. B. Konovalov, V. V. Lyubimov, I. I. Kutuzov, et al., "Application of transform algorithms to high-resolution image reconstruction in optical diffusion tomography of strongly scattering media," Journal of Electronic Imaging, vol. 12, no. 4, pp. 602-612, 2003.

[15] V. V. Lyubimov, O. V. Kravtsenyuk, A. G. Kalintsev, et al., "The possibility of increasing the spatial resolution in diffusion optical tomography," Journal of Optical Technology, vol. 70, no. 10, pp. 715-720, 2003.

[16] O. V. Golubkina, A. G. Kalintsev, A. B. Konovalov, et al., "Application of photon average trajectories method for separate mapping of absorbing and scattering macroinhomogeneities using time-domain measurements technique," in Photon Migration, Optical Coherence Tomography, and Microscopy, vol. 4431 of Proceedings of SPIE, pp. 275-281, Munich, Germany, June 2001.

[17] J. G. Nagy, K. Palmer, and L. Perrone, "Iterative methods for image deblurring: a Matlab object-oriented approach," $\mathrm{Nu}$ merical Algorithms, vol. 36, no. 1, pp. 73-93, 2004.

[18] J. G. Nagy and D. P. O'Leary, "Restoring images degraded by spatially variant blur," SIAM Journal of Scientific Computing, vol. 19, no. 4, pp. 1063-1082, 1998.

[19] J. G. Nagy and D. P. O’Leary, "Fast iterative image restoration with a spatially-varying PSF," in Advanced Signal Processing: Algorithms, Architectures, and Implementations VII, vol. 3162 of Proceedings of SPIE, pp. 388-399, San Diego, Calif, USA, July 1997.

[20] Å. Björck, Numerical Methods for Least Squares Problems, SIAM, Philadelphia, Pa, USA, 1996.

[21] L. Kaufman, "Maximum likelihood, least squares, and penalized least squares for PET," IEEE Transactions on Medical Imaging, vol. 12, no. 2, pp. 200-214, 1993.

[22] J. G. Nagy and Z. Strakos, "Enforcing nonnegativity in image reconstruction algorithms," in Mathematical Modeling, Estimation, and Imaging, vol. 4121 of Proceedings of SPIE, pp. 182190, San Diego, Calif, USA, July 2000.

[23] M. Schweiger, S. R. Arridge, M. Hiraoka, and D. T. Delpy, “The finite element method for the propagation of light in scattering media: boundary and source conditions," Medical Physics, vol. 22, no. 11, pp. 1779-1792, 1995.

[24] D. T. Sandwell, "Biharmonic spline interpolation of GEOS3 and SEASAT altimeter data," Geophysical Research Letters, vol. 14, no. 2, pp. 139-142, 1987.

[25] A. C. Kak and M. Slaney, Principles of Computerized Tomographic Imaging, IEEE Press, New York, NY, USA, 1988.

[26] The Math Work, Using Matlab, Version 6, 2000.

[27] A. Papoulis, Systems and Transforms with Applications in Optics, McGraw-Hills, New York, NY, USA, 1968.

[28] S. Feng, F. Zeng, and B. Chance, "Monte Carlo simulations of photon migration path distributions in multiple scattering media," in Photon Migration and Imaging in Random Media and Tissues, vol. 1888 of Proceedings of SPIE, pp. 78-89, Los Angeles, Calif, USA, January 1993.

[29] S. R. McNown and A. K. Jain, "Approximate shift-invariance by warping shift-variant systems," in The Restoration of HST Images and Spectra II, R. J. Hanisch and R. L. White, Eds., pp. 181-187, Space Telescope Science Institute, Baltimore, Md, USA, 1994.

[30] G. M. Robbins and T. S. Huang, "Inverse filtering for linear shift-variant imaging systems," Proceedings of the IEEE, vol. 60, no. 7, pp. 862-872, 1972.

[31] A. A. Sawchuk, "Space-variant image restoration by coordinate transformations," Journal of the Optical Society of America, vol. 64, no. 2, pp. 138-144, 1974.

[32] H.-M. Adorf, "Towards HST restoration with space-variant PSF, cosmic rays and other missing data," in The Restoration of HST Images and Spectra II, R. J. Hanisch and R. L. White, Eds., pp. 72-78, Space Telescope Science Institute, Baltimore, Md, USA, 1994.

[33] H. J. Trussell and S. Fogel, "Identification and restoration of spatially variant motion blurs in sequential images," IEEE Transactions on Image Processing, vol. 1, no. 1, pp. 123-126, 1992.

[34] D. A. Fish, J. Grochmalicki, and E. R. Pike, "Scanning singularvalue-decomposition method for restoration of images with 
space-variant blur," Journal of the Optical Society of America A: Optics, Image Science, and Vision, vol. 13, no. 3, pp. 464-469, 1996.

[35] J. Kamm and J. G. Nagy, "Kronecker product and SVD approximations in image restoration," Linear Algebra and Its Applications, vol. 284, no. 1-3, pp. 177-192, 1998.

[36] "Restore Tools: An Object Oriented Matlab Package for Image Restoration,” 2002, http://www.mathcs.emory.edu/ nagy/ RestoreTools.

[37] M. Hanke, Conjugate Gradient Type Methods for Ill-Posed Problems, Pitman Research Notes in Mathematics, Longman Scientific \& Technical, Harlow, UK, 1995.

[38] J. G. Nagy and K. M. Palmer, "Steepest descent, CG, and iterative regularization of ill-posed problems," BIT Numerical Mathematics, vol. 43, no. 5, pp. 1003-1017, 2003.

[39] C. R. Vogel, Computational Methods for Inverse Problems, SIAM, Philadelphia, Pa, USA, 2002.

[40] M. Bertero and P. Boccacci, Introduction to Inverse Problems in Imaging, IOP, London, UK, 1998.

[41] W. H. Richardson, "Bayesian-based iterative method of image restoration," Journal of the Optical Society of America, vol. 62, no. 1, pp. 55-59, 1972.

[42] L. B. Lucy, "An iterative technique for the rectification of observed distributions," The Astronomical Journal, vol. 79, no. 6, pp. 745-753, 1974.

[43] G. H. Golub and C. F. van Loan, Matrix Computations, John Hopkins Institute Press, Baltimore, Md, USA, 3rd edition, 1989.

[44] B. M. W. Tsui, X. Zhao, E. C. Frey, and G. T. Gullberg, "Comparison between ML-EM and WLS-CG algorithms for SPECT image reconstruction," IEEE Transactions on Nuclear Science, vol. 38, no. 6, part 2, pp. 1766-1772, 1991.

[45] M. Jiang, G. Wang, M. W. Skinner, J. T. Rubinstein, and M. W. Vannier, "Blind deblurring of spiral CT images," IEEE Transactions on Medical Imaging, vol. 22, no. 7, pp. 837-845, 2003.

[46] C. W. Groetsch, The Theory of Tikhonov Regularization for Fredholm Integral Equations of the First Kind, Pitman, Boston, Mass, USA, 1984.

[47] P. C. Hansen and D. P. O'Leary, "The use of the L-curve in the regularization of discrete ill-posed problems," SIAM Journal on Scientific Computing, vol. 14, no. 6, pp. 1487-1503, 1993.

[48] M. E. Kilmer and D. P. O’Leary, “Choosing regularization parameters in iterative methods for ill-posed problems," SIAM Journal on Matrix Analysis and Applications, vol. 22, no. 4, pp. 1204-1221, 2001.

[49] D. Calvetti, G. Landi, L. Reichel, and F. Sgallari, "Nonnegativity and iterative methods for ill-posed problems," Inverse Problems, vol. 20, no. 6, pp. 1747-1758, 2004.

[50] H. Dehghani, B. W. Pogue, S. P. Poplack, and K. D. Paulsen, "Multiwavelength three-dimensional near-infrared tomography of the breast: initial simulation, phantom, and clinical results," Applied Optics, vol. 42, no. 1, pp. 135-145, 2003.

[51] A. Li, E. L. Miller, M. E. Kilmer, et al., "Tomographic optical breast imaging guided by three-dimensional mammography," Applied Optics, vol. 42, no. 25, pp. 5181-5190, 2003.

[52] J. C. Hebden, S. R. Arridge, and M. Schweiger, "Investigation of alternative data types for time resolved optical tomography," in OSA Technical Digest, Biomedical Topical Meetings, vol. 21, pp. 162-167, Washington, DC, USA, 1998.
Alexander B. Konovalov graduated from the Saint Petersburg State University of Aerospace Instrumentation in electrical engineering in 1987. He is currently a Ph.D. candidate in the Department of Optics and Biomedical Physics, Saratov State University, Saratov. He is now working in the area of image reconstruction as a Senior Research Fellow at the Russian Federal Nuclear Centre, Institute of Technical Physics,

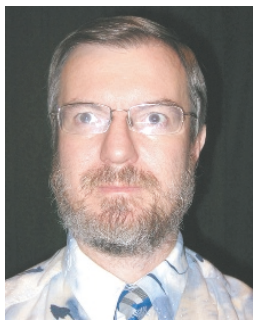
Snezhinsk. He is a Group Leader for Few-View Computed Tomography Group at the same institution. His current research interests include signal and image processing, medical imaging, and biomedical optics. He has authored more than 20 papers in peerrefereed journals and conference proceedings, and more then 30 technical reports for official use only, not open for public.

Vitaly V. Vlasov graduated from the South Ural State University, Chelyabinsk, in applied mathematics, in 2004. He is now a Software Developer at the Russian Federal Nuclear Centre, Institute of Technical Physics, Snezhinsk. He is currently studying towards a Ph.D. degree at the same institution. His research interests include signal and image processing, computer vision, pattern recognition, artificial neural net-

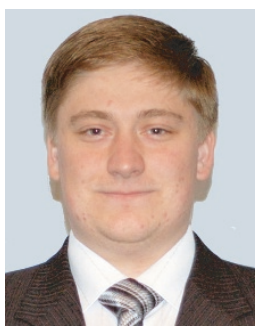
work, visualization, and tomography. He has authored 4 papers in peer-refereed journals and conference proceedings, 5 technical reports for official use only, not open for public.

Olga V. Kravtsenyuk graduated and received her M.S. degree from Saint Petersburg University for Fine Mechanics and Optics in 1996. She received her Ph.D. degree in quantum electronics from the Vavilov State Optical Institute, Saint Petersburg, in 2003. Since 1996, she has been working as a Research Scientist at the Institute for Laser Physics in Saint Petersburg. She is currently occupying a postdoctoral position in Insti-

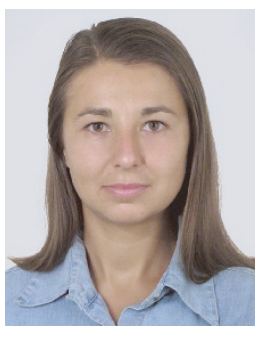
tute of Electronic Structure and Laser, Foundation for Research and Technology, Hellas, Heraklion, Greece, where she is involved in the European Union Project Integrated Technologies for In Vivo Molecular Imaging. She is also a Senior Research Scientist at the Vavilov State Optical Institute. Besides image reconstruction and restoration algorithms for biomedical imaging, her current research interests include biomedical application of optics and lasers.

Vladimir V. Lyubimov graduated from Moscow Physical Technical Institute in physics in 1957. He received his Ph.D. degree in optics from the Vavilov State Optical Institute, Saint Petersburg, in 1971. He is currently a Leading Research Scientist at the Institute for Laser Physics in Saint Petersburg. He is also an Associate Professor in the Department of General Physics at the Saint Petersburg Technological University.

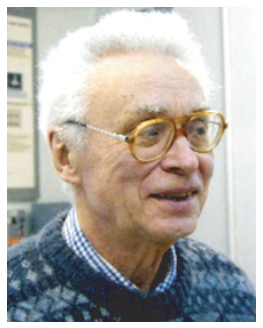

His current research interests include solid-state lasers, laser resonator theory, and diffuse optical tomography. He is currently in the process of submitting a doctoral thesis. 